STRUCTURE REPORTS

ISSN 1600-5368

Received 15 August 2014

Accepted 15 September 2014

Edited by P. Bombicz, Hungarian Academy of Sciences, Hungary

Keywords: crystal structure; benzofuran; 4-fluorophenyl; sulfonyl; hydrogen bonds; $\pi-\pi$ interactions.

CCDC reference: 1021511

Supporting information: this article has supporting information at journals.iucr.org/e

\section{Crystal structure of 2-ethyl-3-(4-fluorophenyl- sulfonyl)-5,7-dimethyl-1-benzofuran}

\author{
Hong Dae Choi ${ }^{\mathrm{a}}$ and Uk Lee ${ }^{\mathrm{b} *}$
}

aDepartment of Chemistry, Dongeui University, San 24 Kaya-dong, Busanjin-gu, Busan 614-714, Republic of Korea, and bepartment of Chemistry, Pukyong National University, 599-1 Daeyeon 3-dong, Nam-gu, Busan 608-737, Republic of Korea. *Correspondence e-mail: uklee@pknu.ac.kr

In the title compound, $\mathrm{C}_{18} \mathrm{H}_{17} \mathrm{FO}_{3} \mathrm{~S}$, the dihedral angle between the plane of the benzofuran ring [r.m.s. deviation $=0.006(1) \AA]$ and that of the 4-fluorophenyl ring [r.m.s. deviation $=0.004(1) \AA$ ] is $82.45(4)^{\circ}$. In the crystal, molecules are linked via three different pairs of $\mathrm{C}-\mathrm{H} \cdots \mathrm{O}$ hydrogen bonds, forming chains along [001] and enclosing two $R_{2}^{2}(10)$ and one $R_{2}^{2}(12)$ ring motifs. The chains are further linked by $\pi-\pi$ interactions [inter-centroid distance $=3.566(1) \AA$ ] between the furan rings of inversion-related molecules, forming a twodimensional network lying parallel to (100).

\section{Chemical Context}

Substituted benzofurans show important pharmacological properties such as antibacterial and antifungal, antitumour and antiviral, and antimicrobial activities (Aslam et al. 2009; Galal et al., 2009; Khan et al., 2005), and are potential inhibitors of $\beta$-amyloid aggregation (Howlett et al., 1999; Ono et al., 2002). These benzofuran compounds occur in a great number of natural products (Akgul \& Anil, 2003; Soekamto et al., 2003). As a part of our ongoing project concerning 2-alkyl3-(phenylsulfonyl)-5,7-dimethyl-1-benzofuran derivatives, we report herein on the synthesis and crystal structure of the title compound.<smiles>CCc1oc2c(C)cc(C)cc2c1S(=O)(=O)c1ccc(F)cc1</smiles>

\section{Structural commentary}

In the title molecule, Fig. 1, the benzofuran unit $(\mathrm{O} 1 / \mathrm{C} 1-\mathrm{C} 8)$ is essentially planar, with a mean deviation of 0.006 (1) Å from the mean plane defined by the nine constituent atoms. The 4fluorophenyl ring (C13-C18) is inclined to the benzofuran ring by $82.45(4)^{\circ}$.

\section{Supramolecular features}

In the crystal, molecules are linked via three different pairs of $\mathrm{C}-\mathrm{H} \cdots \mathrm{O}$ hydrogen bonds, forming chains along [001] and 


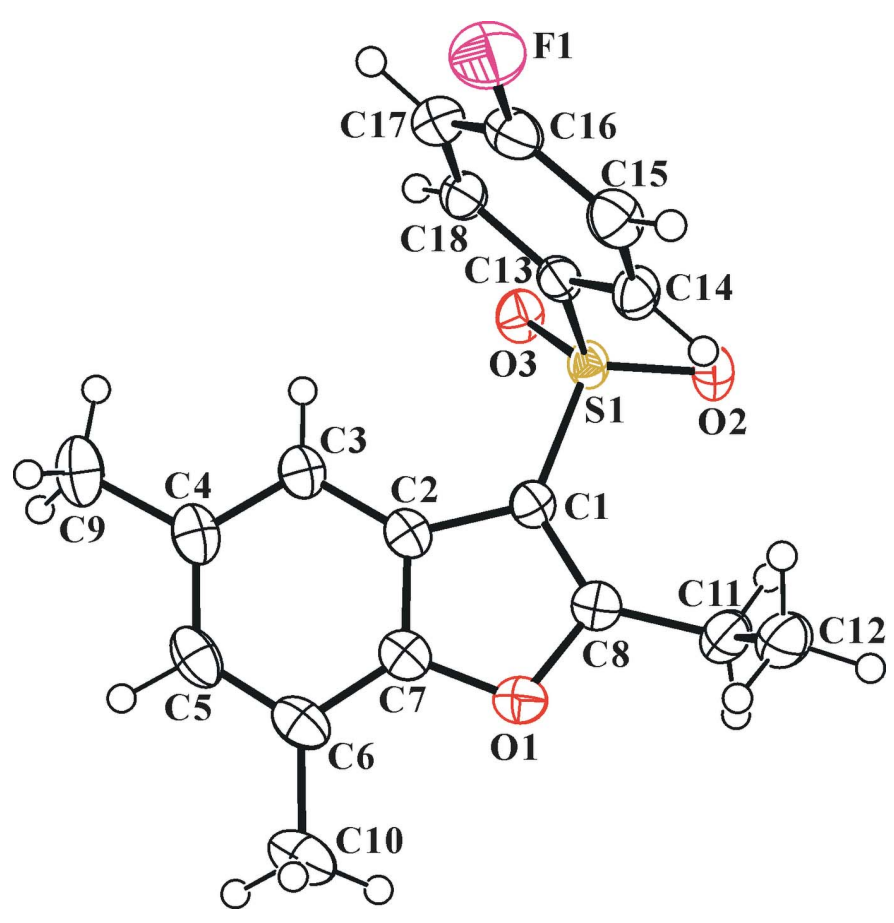

Figure 1

The molecular structure of the title molecule, with atom labelling. Displacement ellipsoids are drawn at the $50 \%$ probability level.

enclosing two $R_{2}^{2}(10)$ and one $R_{2}^{2}(12)$ ring motifs (Fig. 2 and Table 1). The chains are further linked by $\pi-\pi$ interactions between the furan rings of inversion-related molecules, forming a two-dimensional network lying parallel to (100) [illustrated in Fig. 2; $C g 1 \cdots C g 1^{\mathrm{i}}=3.566(1)$, interplanar distance $=3.553(1)$; slippage $=0.305 \AA$; $C g 1$ is the centroid of the $\mathrm{C} 1 / \mathrm{C} 2 / \mathrm{C} 7 / \mathrm{O} 1 / \mathrm{C} 8$ furan ring; symmetry code: (i) $-x+1$, $-y+1,-z+1]$.

\section{Database survey}

A search of the Cambridge Structural Database (Version 5.35, last update May 2014; Allen, 2002) for 3-(phenylsulfonyl)benzofuran gave 65 hits. Six of these involve 5,7-dimethyl-3(phenylsulfonyl)benzofuran derivatives. They include the 2methyl derivative of the title compound, 2-methyl-3-(4fluorophenylsulfonyl)-5,7-dimethyl-1-benzofuran (Choi et al., 2010). In these six compounds, the dihedral angle between the phenylsulfonyl ring and the benzofuran ring varies from $c a$. $72.68^{\circ}$ in the 2-methyl derivative mentioned above, to $87.61^{\circ}$

Table 1

Hydrogen-bond geometry $\left(\AA,^{\circ}\right)$.

\begin{tabular}{lllll}
\hline$D-\mathrm{H} \cdots A$ & $D-\mathrm{H}$ & $\mathrm{H} \cdots A$ & $D \cdots A$ & $D-\mathrm{H} \cdots A$ \\
\hline $\mathrm{C} 3-\mathrm{H} 3 \cdots \mathrm{O} 3^{\mathrm{i}}$ & 0.95 & 2.55 & $3.4804(18)$ & 167 \\
$\mathrm{C} 14-\mathrm{H} 14 \cdots 2^{\mathrm{ii}}$ & 0.95 & 2.49 & $3.1211(17)$ & 124 \\
$\mathrm{C} 18-\mathrm{H} 18 \cdots \mathrm{O}^{\mathrm{i}}$ & 0.95 & 2.36 & $3.2742(17)$ & 160 \\
\hline
\end{tabular}

Symmetry codes: (i) $-x+1,-y+2,-z+1$; (ii) $-x+1,-y+2,-z$.

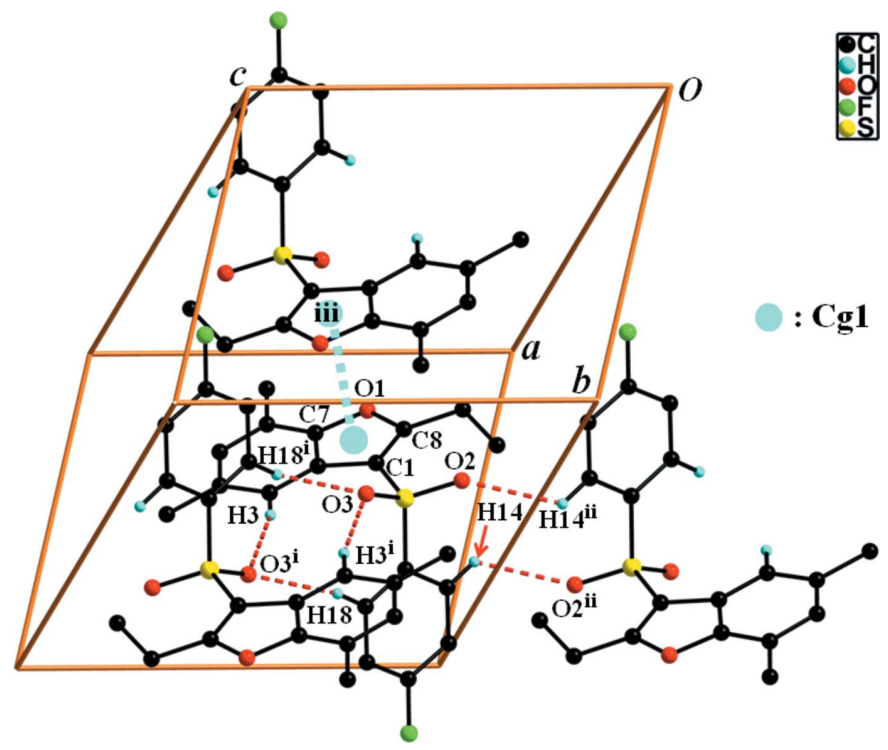

Figure 2

A view of the $\mathrm{C}-\mathrm{H} \cdots \mathrm{O}$ and $\pi-\pi$ interactions (dotted lines) in the crystal structure of the title compound [see Table 1 for details; $\mathrm{H}$ atoms not involved in hydrogen bonding have been omitted for clarity; symmetry codes: (i) $-x+1,-y+2,-z+1$; (ii) $-x+1,-y+2,-z$; (iii) $-x+1$, $-y+1,-z+1]$.

in 2-methyl-3-(2-fluorophenylsulfonyl)-5,7-dimethyl-1-benzofuran (Choi et al., 2014). The same angle in the title compound is $82.45(4)^{\circ}$.

Table 2

Experimental details.

\begin{tabular}{|c|c|}
\hline \multicolumn{2}{|l|}{ Crystal data } \\
\hline Chemical formula & $\mathrm{C}_{18} \mathrm{H}_{17} \mathrm{FO}_{3} \mathrm{~S}$ \\
\hline$M_{\mathrm{r}}$ & 332.38 \\
\hline Crystal system, space group & Triclinic, $P \overline{1}$ \\
\hline Temperature $(\mathrm{K})$ & 173 \\
\hline$a, b, c(\AA)$ & $8.8756(2), 9.3917(2), 11.0284(2)$ \\
\hline$\alpha, \beta, \gamma\left({ }^{\circ}\right)$ & $65.735(1), 80.735(1), 71.145(1)$ \\
\hline$V\left(\mathrm{~A}^{3}\right)$ & $792.68(3)$ \\
\hline$Z$ & 2 \\
\hline Radiation type & Mo $K \alpha$ \\
\hline$\mu\left(\mathrm{mm}^{-1}\right)$ & 0.23 \\
\hline Crystal size (mm) & $0.39 \times 0.33 \times 0.30$ \\
\hline \multicolumn{2}{|l|}{ Data collection } \\
\hline Diffractometer & Bruker SMART APEXII CCD \\
\hline Absorption correction & $\begin{array}{l}\text { Multi-scan (SADABS; Bruker, } \\
\text { 2009) }\end{array}$ \\
\hline$T_{\min }, T_{\max }$ & $0.918,0.936$ \\
\hline $\begin{array}{l}\text { No. of measured, independent and } \\
\text { observed }[I>2 \sigma(I)] \text { reflections }\end{array}$ & $14813,3934,3489$ \\
\hline$R_{\text {int }}$ & 0.025 \\
\hline$(\sin \theta / \lambda)_{\max }\left(\AA^{-1}\right)$ & 0.668 \\
\hline \multicolumn{2}{|l|}{ Refinement } \\
\hline$R\left[F^{2}>2 \sigma\left(F^{2}\right)\right], w R\left(F^{2}\right), S$ & $0.037,0.108,1.07$ \\
\hline No. of reflections & 3934 \\
\hline No. of parameters & 211 \\
\hline $\mathrm{H}$-atom treatment & $\mathrm{H}$-atom parameters constrained \\
\hline$\Delta \rho_{\max }, \Delta \rho_{\min }\left(\mathrm{e} \AA^{-3}\right)$ & $0.29,-0.44$ \\
\hline
\end{tabular}

Computer programs: APEX2 and SAINT (Bruker, 2009), SHELXS97 and SHELXL2014 (Sheldrick, 2008), ORTEP-3 for Windows (Farrugia, 2012), DIAMOND (Brandenburg, 1998) and PLATON (Spek, 2009). 


\section{Synthesis and crystallization}

The starting material 2-ethyl-3-(4-fluorophenylsulfanyl)-5,7dimethyl-1-benzofuran was prepared by a literature method (Choi et al. 1999). 3-Chloroperoxybenzoic acid (77\%, $448 \mathrm{mg}$, $2.0 \mathrm{mmol}$ ) was added in small portions to a stirred solution of 2-ethyl-3-(4-fluorophenylsulfanyl)-5,7-dimethyl-1-benzofuran $(270 \mathrm{mg}, 0.9 \mathrm{mmol})$ in dichloromethane $(35 \mathrm{ml})$ at $273 \mathrm{~K}$. After being stirred at room temperature for $8 \mathrm{~h}$, the mixture was washed with saturated sodium bicarbonate solution $(2 \times$ $15 \mathrm{ml}$ ) and the organic layer was separated, dried over magnesium sulfate, filtered and concentrated at reduced pressure. The residue was purified by column chromatography (hexane-ethyl acetate, $4: 1 \mathrm{v} / \mathrm{v}$ ) to afford the title compound as a colourless solid [yield $61 \%(236 \mathrm{mg})$; m.p. $416-417 \mathrm{~K} ; R_{\mathrm{f}}=$ 0.63 (hexane-ethyl acetate, 4:1 v/v)]. Single crystals suitable for X-ray diffraction were prepared by slow evaporation of a solution of the title compound $(21 \mathrm{mg})$ in acetone $(15 \mathrm{ml})$ at room temperature. ${ }^{1} \mathrm{H}$ NMR $\left(\delta\right.$ p.p.m., $\left.\mathrm{CDCl}_{3}, 400 \mathrm{~Hz}\right)$ : 7.99$8.04(m, 2 \mathrm{H}), 7.47(s, 1 \mathrm{H}), 7.14-7.19(m, 2 \mathrm{H}), 6.93(s, 1 \mathrm{H}), 3.22$ $(q, J=7.52 \mathrm{~Hz}, 2 \mathrm{H}), 2.43(s, 3 \mathrm{H}), 2.41(s, 3 \mathrm{H}), 1.36(t, J=$ $7.54 \mathrm{~Hz}, 3 \mathrm{H})$.

\section{Refinement}

Crystal data, data collection and structure refinement details are summarized in Table 2. All $\mathrm{H}$ atoms were positioned geometrically and refined as riding atoms: $\mathrm{C}-\mathrm{H}=0.95 \AA$ for aryl, $0.99 \AA$ for methylene and $0.98 \AA$ for methyl $\mathrm{H}$ atoms, respectively, with $U_{\text {iso }}(\mathrm{H})=1.5 U_{\text {eq }}(\mathrm{C})$ for methyl $\mathrm{H}$ atoms and $=1.2 U_{\mathrm{eq}}(\mathrm{C})$ for other $\mathrm{H}$ atoms.

\section{Acknowledgements}

The X-ray centre of Gyeongsang National University is acknowledged for providing access to the single-crystal diffractometer.

\section{References}

Akgul, Y. Y. \& Anil, H. (2003). Phytochemistry, 63, 939-943.

Allen, F. H. (2002). Acta Cryst. B58, 380-388.

Aslam, S. N., Stevenson, P. C., Kokubun, T. \& Hall, D. R. (2009). Microbiol.Res. 164, 191-195.

Brandenburg, K. (1998). DIAMOND. Crystal Impact GbR, Bonn, Germany.

Bruker (2009). APEX2, SADABS and SAINT. Bruker AXS Inc., Madison, Wisconsin, USA.

Choi, H. D., Seo, P. J. \& Lee, U. (2014). Acta Cryst. E70, o566.

Choi, H. D., Seo, P. J. \& Son, B. W. (1999). J. Korean Chem. Soc. 43, 606-608.

Choi, H. D., Seo, P. J., Son, B. W. \& Lee, U. (2010). Acta Cryst. E66, o1813.

Farrugia, L. J. (2012). J. Appl. Cryst. 45, 849-854.

Galal, S. A., Abd El-All, A. S., Abdallah, M. M. \& El-Diwani, H. I. (2009). Bioorg. Med. Chem. Lett. 19, 2420-2428.

Howlett, D. R., Perry, A. E., Godfrey, F., Swatton, J. E., Jennings, K. H., Spitzfaden, C., Wadsworth, H., Wood, S. J. \& Markwell, R. E. (1999). Biochem. J. 340, 283-289.

Khan, M. W., Alam, M. J., Rashid, M. A. \& Chowdhury, R. (2005). Bioorg. Med. Chem. 13, 4796-4805.

Ono, M., Kung, M. P., Hou, C. \& Kung, H. F. (2002). Nucl. Med. Biol. 29, 633-642.

Sheldrick, G. M. (2008). Acta Cryst. A64, 112-122.

Soekamto, N. H., Achmad, S. A., Ghisalberti, E. L., Hakim, E. H. \& Syah, Y. M. (2003). Phytochemistry, 64, 831-834.

Spek, A. L. (2009). Acta Cryst. D65, 148-155. 


\section{supporting information}

Acta Cryst. (2014). E70, 249-251 [doi:10.1107/S1600536814019436]

\section{Crystal structure of 2-ethyl-3-(4-fluorophenylsulfonyl)-5,7-dimethyl-1-benzo- furan}

\section{Hong Dae Choi and Uk Lee}

\section{Computing details}

Data collection: APEX2 (Bruker, 2009); cell refinement: SAINT (Bruker, 2009); data reduction: SAINT (Bruker, 2009); program(s) used to solve structure: SHELXS97 (Sheldrick, 2008); program(s) used to refine structure: SHELXL2014 (Sheldrick, 2008); molecular graphics: ORTEP-3 for Windows (Farrugia, 2012) and DIAMOND (Brandenburg, 1998); software used to prepare material for publication: SHELXL2014 (Sheldrick, 2008) and PLATON (Spek, 2009).

\section{2-Ethyl-3-(4-fluorophenylsulfonyl)-5,7-dimethyl-1-benzofuran}

Crystal data

$\mathrm{C}_{18} \mathrm{H}_{17} \mathrm{FO}_{3} \mathrm{~S}$

$M_{r}=332.38$

Triclinic, $P \overline{1}$

Hall symbol: -P 1

$a=8.8756(2) \AA$

$b=9.3917(2) \AA$

$c=11.0284(2) \AA$

$\alpha=65.735(1)^{\circ}$

$\beta=80.735(1)^{\circ}$

$\gamma=71.145(1)^{\circ}$

$V=792.68(3) \AA^{3}$

\section{Data collection}

Bruker SMART APEXII CCD

diffractometer

Radiation source: rotating anode Graphite multilayer monochromator

Detector resolution: 10.0 pixels $\mathrm{mm}^{-1}$ $\varphi$ and $\omega$ scans

Absorption correction: multi-scan (SADABS; Bruker, 2009)

$T_{\min }=0.918, T_{\max }=0.936$

Refinement

Refinement on $F^{2}$

Least-squares matrix: full

$R\left[F^{2}>2 \sigma\left(F^{2}\right)\right]=0.037$

$w R\left(F^{2}\right)=0.108$

$S=1.07$

3934 reflections

211 parameters

$$
\begin{aligned}
& Z=2 \\
& F(000)=348 \\
& D_{\mathrm{x}}=1.393 \mathrm{Mg} \mathrm{m}^{-3} \\
& \text { Melting point }=417-416 \mathrm{~K} \\
& \text { Mo } K \alpha \text { radiation, } \lambda=0.71073 \AA \\
& \text { Cell parameters from } 6147 \text { reflections } \\
& \theta=2.4-28.2^{\circ} \\
& \mu=0.23 \mathrm{~mm}^{-1} \\
& T=173 \mathrm{~K} \\
& \text { Block, colourless } \\
& 0.39 \times 0.33 \times 0.30 \mathrm{~mm}
\end{aligned}
$$

14813 measured reflections 3934 independent reflections 3489 reflections with $I>2 \sigma(I)$

$R_{\text {int }}=0.025$

$\theta_{\max }=28.4^{\circ}, \theta_{\min }=2.0^{\circ}$

$h=-11 \rightarrow 11$

$k=-12 \rightarrow 12$

$l=-14 \rightarrow 14$

0 restraints

Primary atom site location: structure-invariant direct methods

Secondary atom site location: difference Fourier map

Hydrogen site location: difference Fourier map $\mathrm{H}$-atom parameters constrained 
$w=1 /\left[\sigma^{2}\left(F_{\mathrm{o}}^{2}\right)+(0.0587 P)^{2}+0.2229 P\right]$

where $P=\left(F_{\mathrm{o}}^{2}+2 F_{\mathrm{c}}^{2}\right) / 3$

$(\Delta / \sigma)_{\max }<0.001$

$$
\Delta \rho_{\max }=0.29 \mathrm{e} \AA^{-3}
$$$$
\Delta \rho_{\min }=-0.44 \text { e } \AA^{-3}
$$

\section{Special details}

Geometry. All esds (except the esd in the dihedral angle between two 1.s. planes) are estimated using the full covariance matrix. The cell esds are taken into account individually in the estimation of esds in distances, angles and torsion angles; correlations between esds in cell parameters are only used when they are defined by crystal symmetry. An approximate (isotropic) treatment of cell esds is used for estimating esds involving l.s. planes.

Refinement. Refinement of $\mathrm{F}^{2}$ against ALL reflections. The weighted R-factor $\mathrm{wR}$ and goodness of fit $\mathrm{S}$ are based on $\mathrm{F}^{2}$, conventional R-factors $R$ are based on $F$, with $F$ set to zero for negative $F^{2}$. The threshold expression of $F^{2}>2 \operatorname{sigma}\left(\mathrm{F}^{2}\right)$ is used only for calculating R-factors(gt) etc. and is not relevant to the choice of reflections for refinement. R-factors based on $\mathrm{F}^{2}$ are statistically about twice as large as those based on F, and R- factors based on ALL data will be even larger.

Fractional atomic coordinates and isotropic or equivalent isotropic displacement parameters $\left(\hat{A}^{2}\right)$

\begin{tabular}{|c|c|c|c|c|}
\hline & $x$ & $y$ & $z$ & $U_{\text {iso }} * / U_{\text {eq }}$ \\
\hline S1 & $0.47686(4)$ & $0.90767(4)$ & $0.29373(3)$ & $0.02612(11)$ \\
\hline $\mathrm{F} 1$ & $0.95796(13)$ & $1.25062(13)$ & $0.04333(11)$ & $0.0519(3)$ \\
\hline $\mathrm{O} 1$ & $0.69433(12)$ & $0.44317(12)$ & $0.38093(10)$ & $0.0329(2)$ \\
\hline $\mathrm{O} 2$ & $0.37720(12)$ & $0.93598(13)$ & $0.19098(10)$ & $0.0339(2)$ \\
\hline $\mathrm{O} 3$ & $0.40523(12)$ & $0.95254(13)$ & $0.40432(10)$ & $0.0331(2)$ \\
\hline $\mathrm{C} 1$ & $0.58429(16)$ & $0.70342(16)$ & $0.35637(13)$ & $0.0268(3)$ \\
\hline $\mathrm{C} 2$ & $0.68621(15)$ & $0.62616(16)$ & $0.46953(13)$ & 0.0274 \\
\hline $\mathrm{C} 3$ & $0.72804(16)$ & $0.67484(18)$ & $0.55984(13)$ & $0.0300(3)$ \\
\hline H3 & 0.6840 & 0.7834 & 0.5555 & $0.036^{*}$ \\
\hline $\mathrm{C} 4$ & $0.83619(17)$ & $0.5600(2)$ & $0.65658(14)$ & $0.0343(3)$ \\
\hline $\mathrm{C} 5$ & $0.89864(17)$ & $0.40020(19)$ & $0.66131(15)$ & 0.0374 \\
\hline H5 & 0.9724 & 0.3241 & 0.7282 & $0.045^{*}$ \\
\hline C6 & $0.85859(17)$ & $0.34717(18)$ & $0.57367(15)$ & $0.0352(3)$ \\
\hline $\mathrm{C} 7$ & $0.75119(16)$ & $0.46619(17)$ & $0.47913(14)$ & $0.0304(3)$ \\
\hline $\mathrm{C} 8$ & $0.59290(16)$ & $0.58923(17)$ & $0.30738(14)$ & $0.0296(3)$ \\
\hline C9 & $0.8875(2)$ & $0.6073(2)$ & $0.75486(16)$ & $0.0472(4)$ \\
\hline H9A & 0.8299 & 0.7212 & 0.7398 & $0.071^{*}$ \\
\hline H9B & 1.0023 & 0.5946 & 0.7432 & $0.071^{*}$ \\
\hline $\mathrm{H} 9 \mathrm{C}$ & 0.8636 & 0.5370 & 0.8456 & $0.071^{*}$ \\
\hline $\mathrm{C} 10$ & $0.9270(2)$ & $0.17627(19)$ & $0.57832(19)$ & $0.0461(4)$ \\
\hline $\mathrm{H} 10 \mathrm{~A}$ & 0.8523 & 0.1503 & 0.5397 & $0.069^{*}$ \\
\hline H10B & 0.9449 & 0.0998 & 0.6710 & $0.069^{*}$ \\
\hline $\mathrm{H} 10 \mathrm{C}$ & 1.0284 & 0.1673 & 0.5273 & $0.069^{*}$ \\
\hline C11 & $0.52011(18)$ & $0.58876(19)$ & $0.19537(15)$ & $0.0364(3)$ \\
\hline H11A & 0.4181 & 0.6764 & 0.1747 & $0.044^{*}$ \\
\hline H11B & 0.4962 & 0.4836 & 0.2233 & $0.044 *$ \\
\hline $\mathrm{C} 12$ & $0.6270(2)$ & $0.6136(2)$ & $0.06985(16)$ & $0.0425(4)$ \\
\hline $\mathrm{H} 12 \mathrm{~A}$ & 0.6419 & 0.7225 & 0.0361 & $0.064 *$ \\
\hline H12B & 0.5772 & 0.6031 & 0.0020 & $0.064^{*}$ \\
\hline $\mathrm{H} 12 \mathrm{C}$ & 0.7307 & 0.5311 & 0.0907 & $0.064 *$ \\
\hline $\mathrm{C} 13$ & $0.62269(16)$ & $1.01143(16)$ & $0.21923(13)$ & $0.0261(3)$ \\
\hline $\mathrm{C} 14$ & $0.68862(17)$ & $1.01386(17)$ & $0.09555(13)$ & $0.0306(3)$ \\
\hline
\end{tabular}


supporting information

\begin{tabular}{lllll}
$\mathrm{H} 14$ & 0.6562 & 0.9596 & 0.0527 & $0.037^{*}$ \\
$\mathrm{C} 15$ & $0.80202(18)$ & $1.09615(19)$ & $0.03520(14)$ & $0.0348(3)$ \\
$\mathrm{H} 15$ & 0.8485 & 1.0998 & -0.0495 & $0.042^{*}$ \\
$\mathrm{C} 16$ & $0.84549(18)$ & $1.17233(17)$ & $0.10114(15)$ & $0.0349(3)$ \\
$\mathrm{C} 17$ & $0.7805(2)$ & $1.17300(18)$ & $0.22324(15)$ & $0.0363(3)$ \\
$\mathrm{H} 17$ & 0.8130 & 1.2283 & 0.2650 & $0.044^{*}$ \\
$\mathrm{C} 18$ & $0.66666(17)$ & $1.09115(17)$ & $0.28363(14)$ & $0.0307(3)$ \\
$\mathrm{H} 18$ & 0.6194 & 1.0895 & 0.3677 & $0.037^{*}$ \\
\hline
\end{tabular}

Atomic displacement parameters $\left(\AA^{2}\right)$

\begin{tabular}{lllllll}
\hline & $U^{11}$ & $U^{22}$ & $U^{33}$ & $U^{12}$ & $U^{13}$ & $U^{23}$ \\
\hline S1 & $0.02580(17)$ & $0.02980(17)$ & $0.02171(17)$ & $-0.00369(12)$ & $-0.00149(12)$ & $-0.01186(13)$ \\
F1 & $0.0532(6)$ & $0.0496(6)$ & $0.0541(6)$ & $-0.0289(5)$ & $0.0052(5)$ & $-0.0128(5)$ \\
O1 & $0.0315(5)$ & $0.0285(5)$ & $0.0380(5)$ & $-0.0076(4)$ & $0.0000(4)$ & $-0.0131(4)$ \\
O2 & $0.0317(5)$ & $0.0415(6)$ & $0.0287(5)$ & $-0.0063(4)$ & $-0.0073(4)$ & $-0.0145(4)$ \\
O3 & $0.0316(5)$ & $0.0399(5)$ & $0.0275(5)$ & $-0.0046(4)$ & $0.0029(4)$ & $-0.0185(4)$ \\
C1 & $0.0262(6)$ & $0.0289(6)$ & $0.0241(6)$ & $-0.0070(5)$ & $0.0012(5)$ & $-0.0104(5)$ \\
C2 & $0.0243(6)$ & $0.0301(6)$ & $0.0240(6)$ & $-0.0092(5)$ & $0.0027(5)$ & $-0.0069(5)$ \\
C3 & $0.0280(6)$ & $0.0354(7)$ & $0.0243(6)$ & $-0.0101(5)$ & $0.0016(5)$ & $-0.0092(5)$ \\
C4 & $0.0288(7)$ & $0.0445(8)$ & $0.0244(6)$ & $-0.0135(6)$ & $0.0014(5)$ & $-0.0067(6)$ \\
C5 & $0.0288(7)$ & $0.0398(8)$ & $0.0293(7)$ & $-0.0094(6)$ & $-0.0023(6)$ & $0.0008(6)$ \\
C6 & $0.0271(7)$ & $0.0299(7)$ & $0.0371(8)$ & $-0.0084(5)$ & $0.0021(6)$ & $-0.0028(6)$ \\
C7 & $0.0265(6)$ & $0.0307(7)$ & $0.0303(7)$ & $-0.0100(5)$ & $0.0028(5)$ & $-0.0081(5)$ \\
C8 & $0.0266(6)$ & $0.0313(6)$ & $0.0306(7)$ & $-0.0084(5)$ & $0.0018(5)$ & $-0.0124(6)$ \\
C9 & $0.0453(9)$ & $0.0623(11)$ & $0.0312(8)$ & $-0.0132(8)$ & $-0.0081(7)$ & $-0.0144(8)$ \\
C10 & $0.0366(8)$ & $0.0301(7)$ & $0.0568(10)$ & $-0.0065(6)$ & $-0.0028(7)$ & $-0.0042(7)$ \\
C11 & $0.0357(7)$ & $0.0402(8)$ & $0.0403(8)$ & $-0.0100(6)$ & $-0.0021(6)$ & $-0.0226(7)$ \\
C12 & $0.0564(10)$ & $0.0399(8)$ & $0.0350(8)$ & $-0.0134(7)$ & $-0.0004(7)$ & $-0.0187(7)$ \\
C13 & $0.0284(6)$ & $0.0242(6)$ & $0.0225(6)$ & $-0.0029(5)$ & $-0.0030(5)$ & $-0.0086(5)$ \\
C14 & $0.0339(7)$ & $0.0341(7)$ & $0.0252(6)$ & $-0.0077(6)$ & $-0.0014(5)$ & $-0.0139(6)$ \\
C15 & $0.0358(7)$ & $0.0366(7)$ & $0.0279(7)$ & $-0.0081(6)$ & $0.0023(6)$ & $-0.0114(6)$ \\
C16 & $0.0339(7)$ & $0.0274(6)$ & $0.0368(8)$ & $-0.0090(6)$ & $-0.0029(6)$ & $-0.0052(6)$ \\
C17 & $0.0464(8)$ & $0.0290(7)$ & $0.0359(8)$ & $-0.0101(6)$ & $-0.0072(6)$ & $-0.0133(6)$ \\
C18 & $0.0382(7)$ & $0.0281(6)$ & $0.0253(6)$ & $-0.0055(5)$ & $-0.0033(5)$ & $-0.0121(5)$ \\
& & & & & & \\
\hline & & & & & & \\
\hline
\end{tabular}

Geometric parameters $\left(\AA,{ }^{o}\right)$

\begin{tabular}{llll}
\hline $\mathrm{S} 1-\mathrm{O} 2$ & $1.4353(10)$ & $\mathrm{C} 9-\mathrm{H} 9 \mathrm{~B}$ & 0.9800 \\
$\mathrm{~S} 1-\mathrm{O} 3$ & $1.4383(10)$ & $\mathrm{C} 9-\mathrm{H} 9 \mathrm{C}$ & 0.9800 \\
$\mathrm{~S} 1-\mathrm{C} 1$ & $1.7341(14)$ & $\mathrm{C} 10-\mathrm{H} 10 \mathrm{~A}$ & 0.9800 \\
$\mathrm{~S} 1-\mathrm{C} 13$ & $1.7648(14)$ & $\mathrm{C} 10-\mathrm{H} 10 \mathrm{~B}$ & 0.9800 \\
$\mathrm{~F} 1-\mathrm{C} 16$ & $1.3520(17)$ & $\mathrm{C} 10-\mathrm{H} 10 \mathrm{C}$ & 0.9800 \\
$\mathrm{O} 1-\mathrm{C} 8$ & $1.3684(17)$ & $\mathrm{C} 11-\mathrm{C} 12$ & $1.525(2)$ \\
$\mathrm{O} 1-\mathrm{C} 7$ & $1.3833(18)$ & $\mathrm{C} 11-\mathrm{H} 11 \mathrm{~A}$ & 0.9900 \\
$\mathrm{C} 1-\mathrm{C} 8$ & $1.3630(19)$ & $\mathrm{C} 11-\mathrm{H} 11 \mathrm{~B}$ & 0.9900 \\
$\mathrm{C} 1-\mathrm{C} 2$ & $1.4482(18)$ & $\mathrm{C} 12-\mathrm{H} 12 \mathrm{~A}$ & 0.9800 \\
$\mathrm{C} 2-\mathrm{C} 7$ & $1.3892(19)$ & $\mathrm{C} 12-\mathrm{H} 12 \mathrm{~B}$ & 0.9800
\end{tabular}




\begin{tabular}{|c|c|c|c|}
\hline $\mathrm{C} 2-\mathrm{C} 3$ & $1.3935(19)$ & $\mathrm{C} 12-\mathrm{H} 12 \mathrm{C}$ & 0.9800 \\
\hline $\mathrm{C} 3-\mathrm{C} 4$ & $1.392(2)$ & $\mathrm{C} 13-\mathrm{C} 14$ & $1.3891(18)$ \\
\hline $\mathrm{C} 3-\mathrm{H} 3$ & 0.9500 & $\mathrm{C} 13-\mathrm{C} 18$ & $1.3901(19)$ \\
\hline $\mathrm{C} 4-\mathrm{C} 5$ & $1.403(2)$ & $\mathrm{C} 14-\mathrm{C} 15$ & $1.385(2)$ \\
\hline $\mathrm{C} 4-\mathrm{C} 9$ & $1.505(2)$ & $\mathrm{C} 14-\mathrm{H} 14$ & 0.9500 \\
\hline $\mathrm{C} 5-\mathrm{C} 6$ & $1.389(2)$ & $\mathrm{C} 15-\mathrm{C} 16$ & $1.373(2)$ \\
\hline $\mathrm{C} 5-\mathrm{H} 5$ & 0.9500 & $\mathrm{C} 15-\mathrm{H} 15$ & 0.9500 \\
\hline $\mathrm{C} 6-\mathrm{C} 7$ & $1.385(2)$ & $\mathrm{C} 16-\mathrm{C} 17$ & $1.379(2)$ \\
\hline $\mathrm{C} 6-\mathrm{C} 10$ & $1.503(2)$ & $\mathrm{C} 17-\mathrm{C} 18$ & $1.385(2)$ \\
\hline $\mathrm{C} 8-\mathrm{C} 11$ & $1.485(2)$ & $\mathrm{C} 17-\mathrm{H} 17$ & 0.9500 \\
\hline C9-H9A & 0.9800 & $\mathrm{C} 18-\mathrm{H} 18$ & 0.9500 \\
\hline $\mathrm{O} 2-\mathrm{S} 1-\mathrm{O} 3$ & $119.08(6)$ & $\mathrm{C} 6-\mathrm{C} 10-\mathrm{H} 10 \mathrm{~A}$ & 109.5 \\
\hline $\mathrm{O} 2-\mathrm{S} 1-\mathrm{C} 1$ & $108.98(6)$ & $\mathrm{C} 6-\mathrm{C} 10-\mathrm{H} 10 \mathrm{~B}$ & 109.5 \\
\hline $\mathrm{O} 3-\mathrm{S} 1-\mathrm{C} 1$ & $108.06(6)$ & $\mathrm{H} 10 \mathrm{~A}-\mathrm{C} 10-\mathrm{H} 10 \mathrm{~B}$ & 109.5 \\
\hline $\mathrm{O} 2-\mathrm{S} 1-\mathrm{C} 13$ & $108.05(6)$ & $\mathrm{C} 6-\mathrm{C} 10-\mathrm{H} 10 \mathrm{C}$ & 109.5 \\
\hline $\mathrm{O} 3-\mathrm{S} 1-\mathrm{C} 13$ & $107.27(6)$ & $\mathrm{H} 10 \mathrm{~A}-\mathrm{C} 10-\mathrm{H} 10 \mathrm{C}$ & 109.5 \\
\hline $\mathrm{C} 1-\mathrm{S} 1-\mathrm{C} 13$ & $104.43(6)$ & $\mathrm{H} 10 \mathrm{~B}-\mathrm{C} 10-\mathrm{H} 10 \mathrm{C}$ & 109.5 \\
\hline $\mathrm{C} 8-\mathrm{O} 1-\mathrm{C} 7$ & $107.20(11)$ & $\mathrm{C} 8-\mathrm{C} 11-\mathrm{C} 12$ & $113.00(13)$ \\
\hline $\mathrm{C} 8-\mathrm{C} 1-\mathrm{C} 2$ & $108.02(12)$ & $\mathrm{C} 8-\mathrm{C} 11-\mathrm{H} 11 \mathrm{~A}$ & 109.0 \\
\hline $\mathrm{C} 8-\mathrm{C} 1-\mathrm{S} 1$ & $127.10(11)$ & $\mathrm{C} 12-\mathrm{C} 11-\mathrm{H} 11 \mathrm{~A}$ & 109.0 \\
\hline $\mathrm{C} 2-\mathrm{C} 1-\mathrm{S} 1$ & $124.84(10)$ & $\mathrm{C} 8-\mathrm{C} 11-\mathrm{H} 11 \mathrm{~B}$ & 109.0 \\
\hline $\mathrm{C} 7-\mathrm{C} 2-\mathrm{C} 3$ & $119.68(13)$ & $\mathrm{C} 12-\mathrm{C} 11-\mathrm{H} 11 \mathrm{~B}$ & 109.0 \\
\hline $\mathrm{C} 7-\mathrm{C} 2-\mathrm{C} 1$ & $104.35(12)$ & $\mathrm{H} 11 \mathrm{~A}-\mathrm{C} 11-\mathrm{H} 11 \mathrm{~B}$ & 107.8 \\
\hline $\mathrm{C} 3-\mathrm{C} 2-\mathrm{C} 1$ & $135.96(13)$ & $\mathrm{C} 11-\mathrm{C} 12-\mathrm{H} 12 \mathrm{~A}$ & 109.5 \\
\hline $\mathrm{C} 4-\mathrm{C} 3-\mathrm{C} 2$ & $118.14(14)$ & $\mathrm{C} 11-\mathrm{C} 12-\mathrm{H} 12 \mathrm{~B}$ & 109.5 \\
\hline $\mathrm{C} 4-\mathrm{C} 3-\mathrm{H} 3$ & 120.9 & $\mathrm{H} 12 \mathrm{~A}-\mathrm{C} 12-\mathrm{H} 12 \mathrm{~B}$ & 109.5 \\
\hline $\mathrm{C} 2-\mathrm{C} 3-\mathrm{H} 3$ & 120.9 & $\mathrm{C} 11-\mathrm{C} 12-\mathrm{H} 12 \mathrm{C}$ & 109.5 \\
\hline $\mathrm{C} 3-\mathrm{C} 4-\mathrm{C} 5$ & $119.77(15)$ & $\mathrm{H} 12 \mathrm{~A}-\mathrm{C} 12-\mathrm{H} 12 \mathrm{C}$ & 109.5 \\
\hline $\mathrm{C} 3-\mathrm{C} 4-\mathrm{C} 9$ & $120.17(15)$ & $\mathrm{H} 12 \mathrm{~B}-\mathrm{C} 12-\mathrm{H} 12 \mathrm{C}$ & 109.5 \\
\hline $\mathrm{C} 5-\mathrm{C} 4-\mathrm{C} 9$ & $120.05(14)$ & $\mathrm{C} 14-\mathrm{C} 13-\mathrm{C} 18$ & 121.55 \\
\hline $\mathrm{C} 6-\mathrm{C} 5-\mathrm{C} 4$ & $123.62(14)$ & $\mathrm{C} 14-\mathrm{C} 13-\mathrm{S} 1$ & $118.74(10)$ \\
\hline $\mathrm{C} 6-\mathrm{C} 5-\mathrm{H} 5$ & 118.2 & $\mathrm{C} 18-\mathrm{C} 13-\mathrm{S} 1$ & $119.70(10)$ \\
\hline $\mathrm{C} 4-\mathrm{C} 5-\mathrm{H} 5$ & 118.2 & $\mathrm{C} 15-\mathrm{C} 14-\mathrm{C} 13$ & $119.42(13)$ \\
\hline $\mathrm{C} 7-\mathrm{C} 6-\mathrm{C} 5$ & $114.32(14)$ & $\mathrm{C} 15-\mathrm{C} 14-\mathrm{H} 14$ & 120.3 \\
\hline $\mathrm{C} 7-\mathrm{C} 6-\mathrm{C} 10$ & $122.25(15)$ & $\mathrm{C} 13-\mathrm{C} 14-\mathrm{H} 14$ & 120.3 \\
\hline $\mathrm{C} 5-\mathrm{C} 6-\mathrm{C} 10$ & $123.43(14)$ & $\mathrm{C} 16-\mathrm{C} 15-\mathrm{C} 14$ & $118.19(13)$ \\
\hline $\mathrm{O} 1-\mathrm{C} 7-\mathrm{C} 6$ & $125.00(14)$ & $\mathrm{C} 16-\mathrm{C} 15-\mathrm{H} 15$ & 120.9 \\
\hline $\mathrm{O} 1-\mathrm{C} 7-\mathrm{C} 2$ & $110.53(12)$ & $\mathrm{C} 14-\mathrm{C} 15-\mathrm{H} 15$ & 120.9 \\
\hline $\mathrm{C} 6-\mathrm{C} 7-\mathrm{C} 2$ & $124.47(14)$ & $\mathrm{F} 1-\mathrm{C} 16-\mathrm{C} 15$ & $118.29(14)$ \\
\hline $\mathrm{C} 1-\mathrm{C} 8-\mathrm{O} 1$ & $109.89(12)$ & $\mathrm{F} 1-\mathrm{C} 16-\mathrm{C} 17$ & $118.31(14)$ \\
\hline $\mathrm{C} 1-\mathrm{C} 8-\mathrm{C} 11$ & $135.05(13)$ & $\mathrm{C} 15-\mathrm{C} 16-\mathrm{C} 17$ & $123.41(14)$ \\
\hline $\mathrm{O} 1-\mathrm{C} 8-\mathrm{C} 11$ & $115.06(12)$ & $\mathrm{C} 16-\mathrm{C} 17-\mathrm{C} 18$ & $118.51(13)$ \\
\hline $\mathrm{C} 4-\mathrm{C} 9-\mathrm{H} 9 \mathrm{~A}$ & 109.5 & $\mathrm{C} 16-\mathrm{C} 17-\mathrm{H} 17$ & 120.7 \\
\hline $\mathrm{C} 4-\mathrm{C} 9-\mathrm{H} 9 \mathrm{~B}$ & 109.5 & $\mathrm{C} 18-\mathrm{C} 17-\mathrm{H} 17$ & 120.7 \\
\hline $\mathrm{H} 9 \mathrm{~A}-\mathrm{C} 9-\mathrm{H} 9 \mathrm{~B}$ & 109.5 & $\mathrm{C} 17-\mathrm{C} 18-\mathrm{C} 13$ & $118.92(13)$ \\
\hline $\mathrm{C} 4-\mathrm{C} 9-\mathrm{H} 9 \mathrm{C}$ & 109.5 & $\mathrm{C} 17-\mathrm{C} 18-\mathrm{H} 18$ & 120.5 \\
\hline $\mathrm{H} 9 \mathrm{~A}-\mathrm{C} 9-\mathrm{H} 9 \mathrm{C}$ & 109.5 & $\mathrm{C} 13-\mathrm{C} 18-\mathrm{H} 18$ & 120.5 \\
\hline
\end{tabular}




$$
\begin{aligned}
& \mathrm{H} 9 \mathrm{~B}-\mathrm{C} 9-\mathrm{H} 9 \mathrm{C} \\
& \mathrm{O} 2-\mathrm{S} 1-\mathrm{C} 1-\mathrm{C} 8 \\
& \mathrm{O} 3-\mathrm{S} 1-\mathrm{C} 1-\mathrm{C} 8 \\
& \mathrm{C} 13-\mathrm{S} 1-\mathrm{C} 1-\mathrm{C} 8 \\
& \mathrm{O} 2-\mathrm{S} 1-\mathrm{C} 1-\mathrm{C} 2 \\
& \mathrm{O} 3-\mathrm{S} 1-\mathrm{C} 1-\mathrm{C} 2 \\
& \mathrm{C} 13-\mathrm{S} 1-\mathrm{C} 1-\mathrm{C} 2 \\
& \mathrm{C} 8-\mathrm{C} 1-\mathrm{C} 2-\mathrm{C} 7 \\
& \mathrm{~S} 1-\mathrm{C} 1-\mathrm{C} 2-\mathrm{C} 7 \\
& \mathrm{C} 8-\mathrm{C} 1-\mathrm{C} 2-\mathrm{C} 3 \\
& \mathrm{~S} 1-\mathrm{C} 1-\mathrm{C} 2-\mathrm{C} 3 \\
& \mathrm{C} 7-\mathrm{C} 2-\mathrm{C} 3-\mathrm{C} 4 \\
& \mathrm{C} 1-\mathrm{C} 2-\mathrm{C} 3-\mathrm{C} 4 \\
& \mathrm{C} 2-\mathrm{C} 3-\mathrm{C} 4-\mathrm{C} 5 \\
& \mathrm{C} 2-\mathrm{C} 3-\mathrm{C} 4-\mathrm{C} 9 \\
& \mathrm{C} 3-\mathrm{C} 4-\mathrm{C} 5-\mathrm{C} 6 \\
& \mathrm{C} 9-\mathrm{C} 4-\mathrm{C} 5-\mathrm{C} 6 \\
& \mathrm{C} 4-\mathrm{C} 5-\mathrm{C} 6-\mathrm{C} 7 \\
& \mathrm{C} 4-\mathrm{C} 5-\mathrm{C} 6-\mathrm{C} 10 \\
& \mathrm{C} 8-\mathrm{O} 1-\mathrm{C} 7-\mathrm{C} 6 \\
& \mathrm{C} 8-\mathrm{O} 1-\mathrm{C} 7-\mathrm{C} 2 \\
& \mathrm{C} 5-\mathrm{C} 6-\mathrm{C} 7-\mathrm{O} 1 \\
& \mathrm{C} 10-\mathrm{C} 6-\mathrm{C} 7-\mathrm{O} 1 \\
& \mathrm{C} 5-\mathrm{C} 6-\mathrm{C} 7-\mathrm{C} 2 \\
& \mathrm{C} 10-\mathrm{C} 6-\mathrm{C} 7-\mathrm{C} 2 \\
& \mathrm{C} 3-\mathrm{C} 2-\mathrm{C} 7-\mathrm{O} 1 \\
& \mathrm{C} 1-\mathrm{C} 2-\mathrm{C} 7-\mathrm{O} 1
\end{aligned}
$$

109.5

$8.30(15)$
$139.05(12)$
$-106.97(13)$
$-173.96(10)$
$-43.20(13)$
$70.78(12)$
$0.46(14)$
$-177.64(10)$
$-179.96(15)$
$1.9(2)$
$0.70(19)$
$-178.83(14)$
$-0.4(2)$
$178.89(13)$
$-0.1(2)$
$-179.43(14)$
$0.4(2)$
$179.40(14)$
$-179.37(13)$
$0.30(15)$
$179.55(12)$
$0.5(2)$
$-0.1(2)$
$-179.13(13)$
$179.87(11)$
$-0.47(14)$

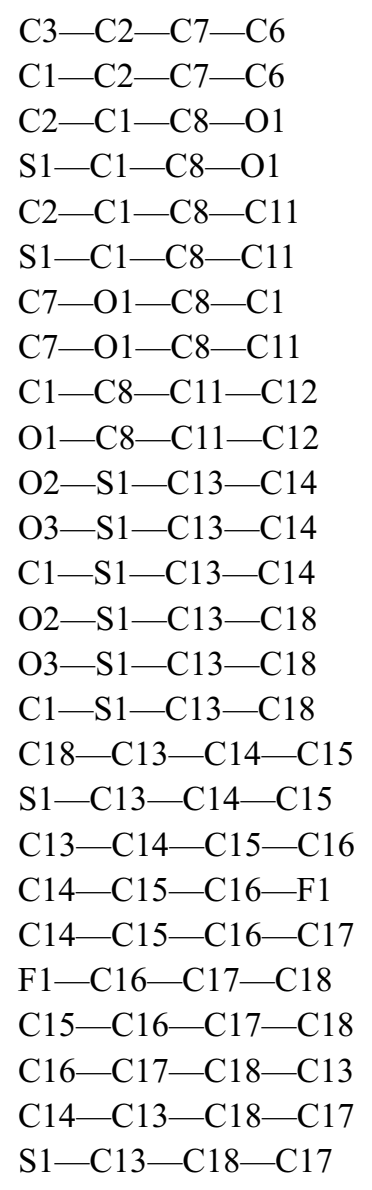

Hydrogen-bond geometry $\left(\AA,{ }^{\circ}\right)$

\begin{tabular}{lllll}
\hline$D-\mathrm{H} \cdots A$ & $D-\mathrm{H}$ & $\mathrm{H} \cdots A$ & $D \cdots A$ & $D-\mathrm{H} \cdots A$ \\
\hline $\mathrm{C} 3-\mathrm{H} 3 \cdots \mathrm{O} 3^{\mathrm{i}}$ & 0.95 & 2.55 & $3.4804(18)$ & 167 \\
$\mathrm{C} 14-\mathrm{H} 14 \cdots 2^{2 i}$ & 0.95 & 2.49 & $3.1211(17)$ & 124 \\
$\mathrm{C} 18-\mathrm{H} 18 \cdots{ }^{\mathrm{i}}{ }^{\mathrm{i}}$ & 0.95 & 2.36 & $3.2742(17)$ & 160 \\
\hline
\end{tabular}

Symmetry codes: (i) $-x+1,-y+2,-z+1$; (ii) $-x+1,-y+2,-z$. 